\title{
Self-Sustained Irregular Activity in an Ensemble of Neural Oscillators
}

\author{
Ekkehard Ullner ${ }^{*}$ and Antonio Politi ${ }^{\dagger}$ \\ Institute for Complex Systems and Mathematical Biology and SUPA, University of Aberdeen, \\ Aberdeen AB24 3UE, United Kingdom
}

(Received 27 August 2015; revised manuscript received 23 November 2015; published 19 February 2016)

An ensemble of pulse-coupled phase oscillators is thoroughly analyzed in the presence of a mean-field coupling and a dispersion of their natural frequencies. In spite of the analogies with the Kuramoto setup, a much richer scenario is observed. The "synchronized" phase, which emerges upon increasing the coupling strength, is characterized by highly irregular fluctuations: A time-series analysis reveals that the dynamics of the order parameter is indeed high dimensional. The complex dynamics appears to be the result of the nonperturbative action of a suitably shaped phase-response curve. Such a mechanism differs from the often-invoked balance between excitation and inhibition and might provide an alternative basis to account for the self-sustained brain activity in the resting state. The potential interest of this dynamical regime is further strengthened by its (microscopic) linear stability, which makes it quite suited for computational tasks. The overall study has been performed by combining analytical and numerical studies, starting from the linear stability analysis of the asynchronous regime, to include the Fourier analysis of the Kuramoto order parameter, the computation of various types of Lyapunov exponents, and a microscopic study of the interspike intervals.

DOI: 10.1103/PhysRevX.6.011015

Subject Areas: Complex Systems,

Nonlinear Dynamics, Statistical Physics

\section{INTRODUCTION}

Most of the challenging questions that arise in the attempt to improve our understanding of the natural (and artificial) world involve multicomponent systems, whose overall dynamics is the result of many nonlinear interactions. The difficulty of the task is often mitigated by the assumption that many phenomena are universal; i.e., they do not crucially depend on the details of the underlying models. It is therefore customary to deal with relatively simple setups in the hopes that relevant details are not missed.

The mammalian brain is the most prominent example where this approach is absolutely necessary if we wish to make some substantial progress. There, even after disregarding several ingredients-such as the multiple degrees of freedom involved in the dynamics of realistic neurons (as in multicompartmental models [1]), the diversity among the single units, the topology, and the plasticity of the connections - the range of possible dynamical phenomena is still very rich and not yet entirely understood. Selfconsistent partial synchronization is a simple but enlightening example. The phenomenon, discovered by van

\footnotetext{
*e.ullner@abdn.ac.uk

†a.politi@abdn.ac.uk
}

Published by the American Physical Society under the terms of the Creative Commons Attribution 3.0 License. Further distribution of this work must maintain attribution to the author(s) and the published article's title, journal citation, and DOI.
Vreeswijk in an ensemble of leaky integrate-and-fire neurons (LIF) [2], was believed, for a long time, to be a nonperturbative effect. However, it has recently been clarified [3] that it is equivalent to the rotating waves observed in the weak-coupling limit [4] and can indeed be observed and characterized in Kuramoto-Daido oscillators [5] as well.

In general, the problem of characterizing the collective dynamics of an ensemble of oscillators is deeply connected to the question of how different levels of descriptions are linked to one another. In computational neuroscience, it is customary to consider subpopulations of neurons, under the assumption that the firing rate is the single relevant variable, as in the seminal paper by Wilson and Cowan [6] and in several other publications (see, e.g., Refs. [1,7,8]). However, it is not clear whether such models can be derived starting from more microscopic setups based on single spiking neurons. Some recent studies have shown that a low-dimensional collective dynamics may emerge in networks of theta (or, equivalently, quadratic-integrate-and-fire, QIF) neurons $[9,10]$. More than that, a reformulation of pulse-coupled oscillators in terms of firing-rate models has been accomplished in Refs. [11,12]. The validity of these results is due to the existence of relationships such as the Ott-Antonsen Ansatz [13] and the Watanabe-Strogatz theorem [14], which allow one to express the collective behavior in terms of a few variables, the others being essentially slaved. Such theoretical pillars are, however, based on strong simplifying assumptions on the nature of the interoscillator coupling $[13,14]$.

To what extent is the compression of degrees of freedom effective in more general setups? The background activity 
of the brain in the resting state, when no specific task is performed [15-17], testifies to a collective irregular dynamics. Moreover, the ongoing discussion about rate versus temporal coding $[18,19]$ suggests that the firing rate may not be sufficient to ensure the necessary computational capability of the mammalian brain.

Altogether, one should thus expect an irregular collective behavior. It is often conjectured that the self-sustained activity is the result of a balance between activation and inhibition [20,21]. Mathematically, this means that the effect of the coupling is zero on average, so it is essentially controlled by stochastic or chaotic fluctuations. However, it is not clear how such a balance can be durably ensured in self-organized networks of firing oscillators. A conceptually different possibility to account for a macroscopic irregularity is offered by the nonlinear character of the Liouville-type equation (which, strictly speaking, applies to an ensemble of infinitely many oscillators). This functional equation operates in an infinite-dimensional phase space and can, thereby, generate a dynamics of arbitrary complexity. This result has indeed been observed in an abstract model of coupled maps [22] and in globally coupled StuartLandau oscillators [23]. In both cases, the single dynamical units are intrinsically more complex than phase oscillators: The logistic maps are chaotic by themselves, Stuart-Landau oscillators can behave chaotically under the action of a periodic modulation. In the case of phase oscillators, there is only preliminary evidence in an ensemble of LIF neurons with delayed interactions [24].

In this paper, we present a model whose overall activity is intrinsically high dimensional. As this dynamical phase is rather robust against variations of several parameters, it may provide an alternative mechanism for the selfsustainment of the resting brain activity. More precisely, we study an ensemble of pulse-coupled phase oscillators, whose phase-response curve (PRC) is derived by smoothing the PRC of LIF neurons. Other than that, our setup is the same as in the standard Kuramoto model $[25,26]$ : The single oscillators are characterized by a distribution of bare frequencies, while the coupling is homogeneously all-toall. As in the Kuramoto model, a synchronization transition is observed upon increasing the coupling strength, but the analogies end here since, above criticality, the order parameter, rather than being constant, exhibits complex high-dimensional oscillations.

As briefly discussed in Sec. III, in the weak-coupling limit (and for a small dispersion of the frequencies), our system reduces to a Kuramoto-Daido model, with a coupling function composed of several Fourier harmonics. Recent studies have revealed that such a model exhibits a rich phenomenology (see, e.g., Refs. [27-29]). For instance, in the presence of a second harmonic, a high degree of multistability is observed, with many different cluster states being simultaneously stable, termed "multibranch entrainment" $[27,28,30,31]$. However, no stationary irregular collective dynamics has been observed so far (the order parameter exhibits, at most, regular periodic oscillations). The only mechanism that has been found to trigger macroscopic fluctuations is the presence of two distinct populations [32], and yet the oscillations are a manifestation of lowdimensional chaos. In our setup, a single population of oscillators is instead able to exhibit high-dimensional chaotic dynamics. The macroscopic fluctuations are present in the "activity" field as well, i.e., in the sum of all spikes emitted by the neurons. This variable is akin to the electric potential recorded while measuring EEGs.

Another feature that makes the observed scenario strikingly different is the existence of a fully negative spectrum of Lyapunov exponents. This inconsistency is nothing but a manifestation of stable chaos [33], an irregular dynamics of cellular-automaton type, which is self-sustained because of the high (infinite) dimensionality of the phase space (in other words, it dies out in finite ensembles). In neural systems, stable chaos was first found in a diluted network of LIF units [34] and later discussed in more disordered setups [35,36]. At variance with deterministic chaos, accompanied by an exponential separation of orbits and thereby a loss of memory, stable chaos is identified by a "microscopically" stable dynamics, which is definitely more appropriate for the performance of computational tasks. The potentiality of stable chaos for information processing has been preliminarily explored in Refs. [37,38]. The onset of a macroscopic irregular dynamics, as discussed in this paper, makes this perspective even more intriguing for the richness of the collective behavior.

In Sec. II, we introduce the setup and justify its choice. In Sec. III, we reconstruct the asynchronous state and investigate its stability properties. Differences and analogies with the standard Kuramoto model are emphasized. In particular, we find that the asynchronous regime loses its stability when a complex eigenvalue is born out of the line containing the continuous spectrum. In the last part of the section, the proper order parameters for the characterization of the transition are introduced: They are the Kuramoto order parameter, whose definition requires passing to more appropriate phase variables, and the activity field. Sections IV and V are devoted to a careful numerical analysis of the synchronized phase at the collective and microscopic levels, respectively. Because of the difficulty of dealing with finite-size corrections, we study the resulting behavior sufficiently far from the transition. In Sec. IV, we first illustrate the phase diagram and the initial part of the Lyapunov spectrum. We then show the power spectrum of the order parameter and carry on a time-series analysis to determine the fractal dimension. In Sec. V, we focus our interest on the behavior of the single neurons, computing the effective frequency and the conditional Lyapunov exponents: They are all negative, indicating that we are in the presence of generalized synchronization. The presence of phase slips is also unveiled. Finally, in the last 
section, we summarize the main results and discuss the several perspectives that are opened by the scenario discussed in the paper.

\section{THE MODEL}

The starting point of this paper is the model of delayed LIF neurons studied in Ref. [24]. Here, the model is modified to make it simpler, more generic, and more amenable to both numerical and analytical studies.

In this perspective, we consider an ensemble of pulsecoupled phase oscillators in the presence of $\delta$-like pulse and characterized by a suitable PRC $\Gamma(\phi)$,

$$
\dot{\phi}_{i}=\omega_{i}-\frac{g}{N} \Gamma\left(\phi_{i}\right) \sum_{j} \delta\left(t-t_{j}\right)
$$

where $\phi_{i} \in[0,1]$ is the local phase, $\omega_{i}$ the bare oscillator frequency (i.e., in the absence of coupling), $g$ the coupling strength, and $N$ the system size. Whenever any oscillator reaches the threshold $\phi_{i}=1$, a $\delta$ spike is sent and received by all neurons. The above formulation is quite general for two reasons: (i) Any model where the velocity field $\dot{\phi}_{i}$ is phase dependent (in the absence of coupling) can always be rephrased as Eq. (1) upon suitably changing variables [39]; (ii) finite-width pulses can be mapped onto $\delta$-like ones upon suitably adjusting the shape of the PRC [3] (at least in the weak-coupling limit).

An important ingredient of the model studied in Ref. [24] is the presence of a delay between spike emission and reception. In the weak-coupling limit, when the dynamics is nearly homogeneous, one can simulate the presence of a delay as a suitable phase shift of the PRC, and this is what has been assumed here. The phase shift should be different for the different oscillators. However, here, for the sake of simplicity, we assume the same PRC for all the oscillators.

In the LIF model, $\Gamma\left(\phi_{i}\right)=a \exp \left(b \phi_{i}\right)$, where $\phi_{i}$ is assumed to be taken modulus 1 . As a result of the phase shift, the discontinuity originally present when passing from 1 to 0 moves inside the unit interval. For the sake of generality and simplicity, we prefer to remove the discontinuity, considering a piecewise linear PRC, such as

$$
\Gamma(\phi)= \begin{cases}B_{01}+b_{1} \phi & \text { if } 0 \leq \phi<\phi_{l} \\ B_{02}-b_{2} \phi & \text { if } \phi_{l} \leq \phi \leq \phi_{r} \\ B_{03}+b_{1} \phi & \text { if } \phi_{r}<\phi<1,\end{cases}
$$

where the various parameters are chosen so as to ensure continuity in $\phi_{l}, \phi_{r}$ and equality between $\phi=0$ and 1 . Considering that the amplitude of the PRC is controlled by the coupling constant $g$, there are three truly independent parameters: one controlling the vertical shift of the PRC, and two that identify the junction points. As for the first parameter, it basically controls whether the coupling has an average excitatory or inhibitory effect, thereby inducing a speeding up or slowing down of the spiking activity. Since we are not interested in such effects, but rather in the mutual attraction or repulsion among the oscillators, we have decided to assume that the PRC has zero average. The two remaining parameters are identified by the phase shift $s$ (defined as the distance of the midpoint of the central region from 1 -see Fig. 1) and the width $\delta$ of the central interval. Altogether, $b_{2}=b_{1} / \delta, \quad B_{01}=b_{1}(s-1 / 2)$, $B_{02}=b_{1}(1-s) / \delta$, and $B_{03}=b_{1}(s-3 / 2)$, while $\phi_{l}=$ $(1-s+\delta / 2-\delta s) /(\delta+1)$ and $\phi_{r}=(1-s+3 \delta / 2-\delta s) /$ $(\delta+1)$. The parameter $b_{1}$ has been set equal to 1.5 (in principle, it can be absorbed in the definition of $g$ ), while the two other parameters have been set as $s=0.14, \delta=0.1$ in all of the following simulations. The resulting shape of the PRC is presented in Fig. 1.

Finally, we have chosen to work with a uniform distribution of frequencies $P(\omega)$ centered in $\bar{\omega}=1.4$. The simulations reported in this paper refer to a width $\Delta=\left(\omega_{\max }-\omega_{\min }\right)=1.2$, but similar results have been obtained for different values of $\Delta$. We evolve the model, Eqs. (1) and (2), as an event-driven process. Between two consecutive $\delta$ spikes, the phase of each oscillator increases linearly according to its individual bare frequency $\omega_{i}$. When one of the oscillators reaches the firing threshold $\phi_{i}=1$, its phase is reset to zero and all phases are adjusted to account for the received spike. The effect of the coupling might bring a second oscillator beyond the firing threshold. In such a case, that oscillator is also reset to zero plus an offset because of the spike received from the first oscillator. We continue this evolution, without advancing the time, until no further spikes are triggered. In practice, "avalanches" may occur: They do not contribute significantly to the global behavior as their size does not increase upon increasing the number of neurons. Notice also that in the original model [24], avalanches do not exist.

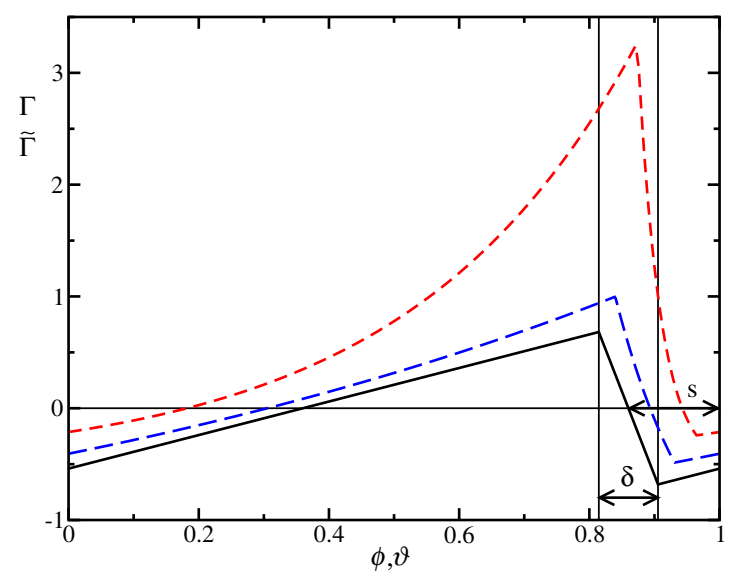

FIG. 1. Phase response curve $\Gamma\left(\phi_{i}\right)$ according to Eq. (2) for the standard parameter values $b_{1}=1.5, s=0.14$, and $\delta=0.1$ (solid line). The short- and long-dashed curves correspond to the effective PRC $\tilde{\Gamma}$ [see Eq. (13) and corresponding text] obtained for $\omega=\omega_{\min }=0.8$ and $\omega=\omega_{\max }=2$, respectively (with $g=0.8)$. 


\section{THEORY}

Some insight can be gained by considering the thermodynamic limit, as this allows us to analytically determine the properties of the stationary asynchronous regime.

It is convenient to define the activity field $E(t)$ as the number of spikes emitted per unit time, so Eq. (1) can be rewritten as (for the sake of simplicity, we drop the subindex $i$ )

$$
\dot{\phi}=\omega-g \Gamma(\phi) E(t) .
$$

Let us now introduce the probability density $Q(\phi, \omega, t)$, as the fraction of neurons with a bare frequency in $[\omega, \omega+d \omega)$, whose phase belongs to $[\phi, \phi+d \phi)$ at time t. Clearly,

$$
\int Q(\phi, \omega, t) d \phi=P(\omega),
$$

where $P(\omega)$ is the time-independent density of neurons with bare frequency $\omega$ defined in the previous section. $Q$ satisfies the continuity equation

$$
\frac{\partial Q}{\partial t}=-\frac{\partial}{\partial \phi}[\omega-g \Gamma(\phi) E(t)] Q,
$$

while the field $E$ satisfies the self-consistent equation

$$
E(t)=\int Q(1, \omega, t)[\omega-g \Gamma(1) E(t)] d \omega,
$$

which implies

$$
E(t)=\frac{\int \omega Q(1, \omega, t) d \omega}{1+g \Gamma(1) \int Q(1, \omega, t) d \omega} .
$$

The asynchronous regime corresponds to the stationary solution and is thereby characterized by a constant mean field. The phase dependence of $Q(\phi, \omega, t)$ can be determined by setting its time derivative equal to zero. By properly renormalizing the flux, one obtains

$$
Q_{0}(\phi, \omega)=\frac{P(\omega)}{T(1, \omega)\left[\omega-g \Gamma(\phi) E_{0}\right]},
$$

where

$T\left(\psi, \omega, E_{0}\right)=\int_{0}^{\psi} \frac{d \phi}{\omega-g \Gamma(\phi) E_{0}} \equiv \int_{0}^{\psi} \tau(\phi, \omega) d \phi$

is the time required by an oscillator with frequency $\omega$ to reach the phase $\psi$, starting from 0 , in the presence of a constant field $E_{0} . T\left(1, \omega, E_{0}\right)$ is thereby the interspike interval, while $\tau\left(\phi_{i}, \omega\right)$ is the inverse instantaneous effective frequency. The field $E_{0}$ can finally be obtained from Eqs. (5) and (6),

$$
E_{0}=\int \frac{P(\omega)}{T(1, \omega)} d \omega .
$$

The above calculation yields the structure of the asynchronous state, but it does not tell us whether it is stable. The stability can be assessed by investigating the behavior of infinitesimal perturbations. Let us define

$$
\begin{aligned}
Q(\phi, \omega, t) & =Q_{0}(\phi, \omega)+q(\phi, \omega, t), \\
E(t) & =E_{0}+e(t),
\end{aligned}
$$

where $q$ and $e$ satisfy the following equations,

$$
\frac{\partial q}{\partial t}=-\frac{\partial}{\partial \phi}\left[\omega-g \Gamma(\phi) E_{0}\right] q+g e(t) \frac{\partial \Gamma(\phi) Q_{0}}{\partial \phi}
$$

and

$$
e(t)=\frac{\int\left(\omega-g \Gamma(1) E_{0}\right) q(1, \omega, t) d \omega}{1+g \Gamma(1) \int Q_{0}(1, \omega) d \omega} .
$$

These two equations can be solved by introducing a standard Ansatz, $\quad q(\phi, \omega, t)=u(\phi, \omega) \mathrm{e}^{\mu t}, \quad e(t)=z \mathrm{e}^{\mu t}$. One obtains

$$
\mu u=g \Gamma^{\prime} E_{0} u-\left[\omega-g \Gamma E_{0}\right] u^{\prime}+g \Gamma^{\prime} Q_{0} z+g \Gamma Q_{0}^{\prime} z
$$

and

$$
z=\frac{\int\left(\omega-g \Gamma(1) E_{0}\right) u(1, \omega) d \omega}{1+g \Gamma(1) \int Q_{0}(1, \omega) d \omega},
$$

where the prime denotes a derivative with respect to $\phi$ and we have dropped the dependence on $\phi$ for simplicity of presentation. The solution of such an equation, reported in Appendix A, yields the eigenvalue equation (A9).

The spectrum of the linear operator consists of a continuous and a discrete component. The continuous part is confined to an interval along the imaginary axis and is therefore composed of marginally stable directions. The discrete component can be obtained by assuming $\mu=\mu_{R}+i \mu_{I}$, separating Eq. (A9) into real and imaginary parts, and finally looking for the zeros in the complex plane.

A numerical study reveals the presence of (at least) three pairs of complex-conjugate eigenvalues (see Fig. 2, where the imaginary part is not reported), two of which are negative and one which is positive. Two pairs of exponents arise definitely above some finite $g$ value; the third one is likely to follow the same scenario, but given its small real part, we could not trace it for small coupling strengths (see the crosses in Fig. 2). Altogether, the asynchronous solution is marginally stable up to $g_{c} \approx 0.72$, when it destabilizes for the onset of a pair of complex-conjugate eigenvalues with a positive real part. 


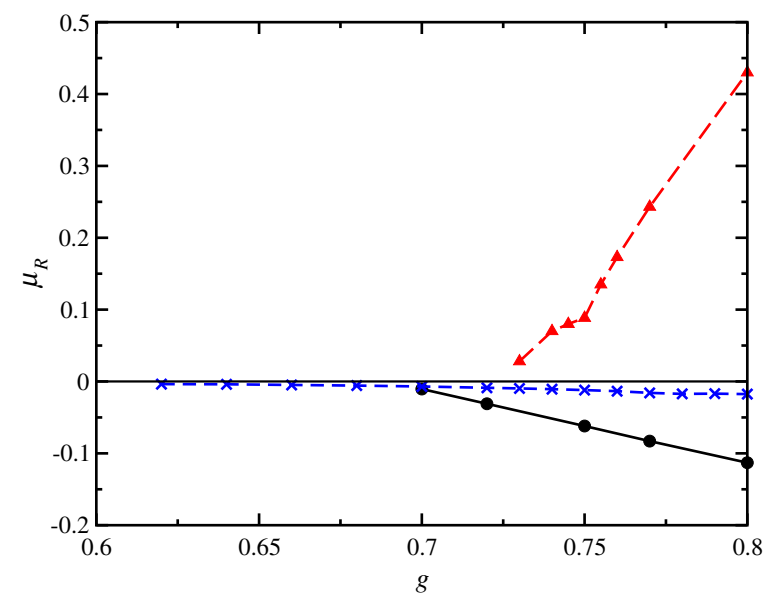

FIG. 2. Stability diagram of the asynchronous state. The real part of the discrete eigenvalues is reported versus the coupling strength $g$.

Let us now compare our findings with the stability of the asynchronous solution in the Kuramoto model. Below criticality, in both cases, the probability distribution is marginally stable (see Ref. [40] for the first such analysis in the Kuramoto setup), the major difference being the presence of a discrete stable spectral component in our model. It is noteworthy that, in spite of the marginal stability of the probability density, the order parameter (see the next section for its definition) relaxes exponentially in the Kuramoto model. This is a manifestation of the socalled Landau damping [41]. Only recently has this "inconsistency" been fully resolved by understanding that different classes of functions may be considered in the stability analysis [42-44]. We do not know how much of such studies carry over to the present setup: This is an open problem.

At criticality, a pair of complex eigenvalues with a positive real part is born: This is at variance with the standard Kuramoto model, where the newly appearing eigenvalue is real. There is, instead, an analogy with the Kuramoto model with delay $[45,46]$, where periodic oscillations arise. Here, however, above threshold, the probability density, rather than oscillating periodically, behaves irregularly, as discussed in the following sections.

\section{A. Order parameters}

The onset of synchronization is typically studied by using the distribution of phases to determine the Kuramoto order parameter [25]. However, here, one cannot directly use the phase $\phi$ since it does not advance homogeneously in the asynchronous regime. However, assuming a stationary field $E_{0}$ (this is the signature of the asynchronous regime), one can define a phase $\vartheta$ such that it increases linearly with an effective frequency $\tilde{\omega}=1 / T\left(1, \omega, E_{0}\right)$. From Eq. (3), it is easily seen that this can be achieved by assuming that $[3,39]$

$$
\frac{d \vartheta}{d \phi}=\frac{\tilde{\omega}}{\omega-g \Gamma(\phi) E_{0}} .
$$

This new phase follows the local dynamics and is described by the equation

$$
\dot{\vartheta}=\tilde{\omega}-g \tilde{\Gamma}(\vartheta)\left(E(t)-E_{0}\right),
$$

where $\tilde{\Gamma}(\vartheta)$ is the effective PRC

$$
\tilde{\Gamma}(\vartheta)=\frac{\tilde{\omega} \Gamma(\phi(\vartheta))}{\omega-g E_{0} \Gamma(\phi(\vartheta))},
$$

and $\vartheta(\phi)$ is obtained by solving Eq. (11). As understood from its definition, $\vartheta(\phi)$ depends both on $g$ and the bare frequency. The dependence of $\vartheta$ on $\phi$ is reported in Fig. 3 for the maximal and minimal frequencies at $g=0.8$. The particular transformation from the phase $\phi$ to the new effective phase $\vartheta$ is shown in Appendix B.

Once the proper phase $\vartheta$ has been identified, a whole series of generalized order parameters can be defined [5],

$$
R_{k}=\frac{1}{N}\left|\sum_{j} \mathrm{e}^{2 \pi i k \vartheta_{j}}\right| \text { with } k \geq 1,
$$

which are nothing but the Fourier modes of the phase probability density. The standard Kuramoto order parameter $R_{1}$ [25] is typically used to monitor the onset of synchronization (for the sake of simplicity, in the following, the subscript " 1 " is typically dropped). Higher-order parameters, all equal to zero in the asynchronous regime, help to provide a more accurate characterization of the phase distribution.

Given the complexity of the transformations needed to determine $R$, and because of the relationship with neural networks, we have often considered another order parameter, a smoothened version $Y(t)$ of the field $E(t)$. In a finite

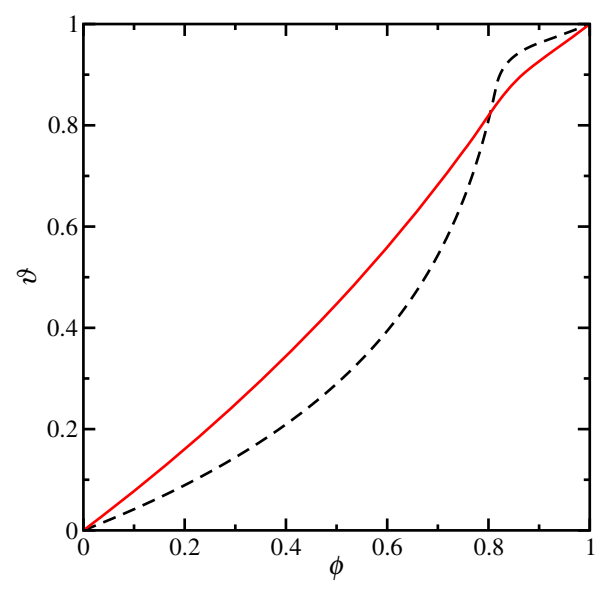

FIG. 3. $\vartheta(\phi)$ for $g=0.8$ and two different frequencies: $\omega=0.8$ (dashed curve) and $\omega=2$ (solid curve). 
system, $E(t)$ is just a collection of $\delta$ pulses. It is therefore more convenient to investigate

$$
\dot{Y}=-\gamma Y+E(t) \text {. }
$$

We have selected $\gamma=5$. In the asynchronous regime, the activity is constant, i.e., $Y_{0}=E_{0} / \gamma$. Above the transition that we are going to discuss, the activity starts oscillating in time, so it is convenient to introduce the temporal standard deviation

$$
\sigma_{Y}=\sqrt{\left\langle Y^{2}\right\rangle-\langle Y\rangle^{2}},
$$

where the angular brackets denote a time average. It will also be useful to look at the fluctuation $\sigma_{R}$ of the Kuramoto order parameter $R$, as this indicator allows us to identify the regimes where the degree of synchronization oscillates in time.

We end this theoretical section by briefly commenting on the weak-coupling, low-disorder limit. The effective PRC $\tilde{\Gamma}$ depends on the frequency of the oscillator (and on the coupling strength). The resulting curves for $\omega_{\min }$ and $\omega_{\max }$ (and $g=0.8$ ) are reported in Fig. 1: see the dashed lines. For small frequency dispersion, the use of averaging techniques suggests that the model becomes equivalent to the Kuramoto-Daido model (see Ref. [47] for a general treatment and Ref. [3] for a quantitative analysis of a much similar setup),

$$
\dot{\vartheta}_{i}=\omega_{i}-\frac{g}{N} \sum_{j} \tilde{\Gamma}\left(\vartheta_{i}-\vartheta_{j}\right)
$$

The above equation makes one difference with the Kuramoto model transparent: The sinusoidal coupling function is replaced by the more structured function $\tilde{\Gamma}$ (see Fig. 1). None of the several simulations performed with small coupling and small frequency dispersion, however, has been able to reproduce the complex scenario discussed in this paper.

\section{MACROSCOPIC DYNAMICS}

The most appropriate control parameter to study the onset of collective dynamics is the coupling strength $g$. In Figs. 4(a) and 4(b), it is used to parametrize the dependence of the Kuramoto order parameter $R$, its temporal standard deviation $\sigma_{R}$, and the standard deviation of the activity field $Y$.

Each data point is based on a simulation over 500 time units after a transient of 50 time units. All but the red curve have been obtained by increasing the coupling strength $g$ stepwise, using the final condition for a given $g$ value as the initial condition for the next one.

The simulations performed for three different system sizes (4000, 16000, and 64000 units) reveal the existence of

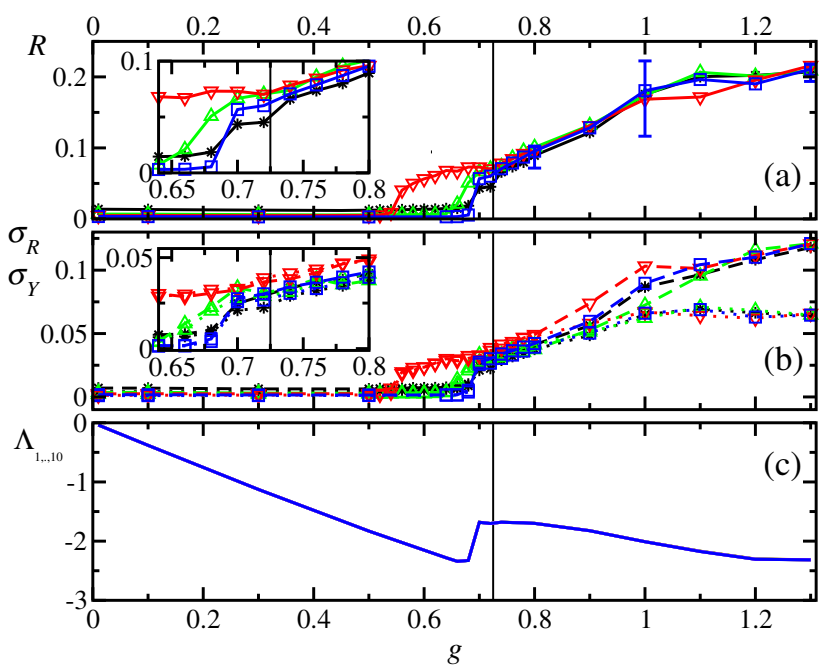

FIG. 4. Phase diagram: Dependence of the Kuramoto order parameter $R(\mathrm{a})$, its standard deviation $\sigma_{R}$, the standard deviation $\sigma_{Y}$ of the activity field $Y$ (b), and the ten largest Lyapunov exponents (c) versus the coupling strength $g$. The curves in (a) and (b) have been obtained for $N=4000$ (black), $N=16000$ (green), and $N=64000$ (blue), upon increasing the coupling $g$. The red curve ( $\mathrm{a}$ and $\mathrm{b}$ ) is a continuation by decreasing $g$ with $N=64000$, and $\sigma_{R}$ and $\sigma_{Y}$ are represented with dashed and dotted lines, respectively (b). The vertical lines mark the critical point $g_{c} \approx 0.72$, where the linear stability of the asynchronous state is lost (see Sec. III). The insets in panels (a) and (b) reveal the zone of the fuzzy transition. The ten largest global Lyapunov exponents in panel (c) are almost indistinguishable. The simulations have been performed for $N=64000$, increasing $g$. We introduce and discuss the global Lyapunov exponent in the last paragraph of this section.

a critical coupling value $g_{c}$ above which $R$ grows from zero. At variance with the Kuramoto model, the standard deviation $\sigma_{R}$ is larger than zero above $g_{c}$, meaning that the degree of synchronization fluctuates. The onset of macroscopic oscillations is confirmed by the behavior of the other order parameter $\sigma_{Y}$. The critical value is close to $g_{c}$ as estimated from the linear stability analysis discussed in the previous section. A quantitative conclusion is, however, problematic. The green curve in Fig. 4, which refers to $N=16000$, deviates at criticality from those obtained for both smaller (4000) and larger (64000) system sizes. On the one hand, simulations performed for different realizations of the bare frequencies show that such deviations might just be sample-to-sample fluctuations. On the other hand, we cannot exclude the fact that we are before a "fuzzy" transition, i.e., a finite interval of the control parameter, where the collective dynamics varies in an irregular way, as found in a chain of coupled maps exhibiting stable chaos [48].

Besides sample-to-sample fluctuations, the measurements are obviously affected by statistical uncertainty as well. Such an error has been estimated by dividing the standard deviation of $R$ by the square root of the number $N_{e}$ 
of effectively independent time intervals and $N_{e}$, determined as the ratio between the total length of the time series by the decay time of the autocorrelation function. This is typically very small except for $g \approx 1$ (see the error bars), where the correlation decays quite slowly.

The red curve tracks the mean-field obtained by decreasing $g$. The difference observed in the critical region with respect to the previous curves (obtained by increasing $g$ ) suggests the possible coexistence of an asynchronous regime with a partially synchronized regime. Since, however, no jump is observed in the simulations performed by increasing $g$, it is reasonable to conclude that the bifurcation is "supercritical" and thus to attribute such deviations to the finite sweeping time. Since the main goal of this work is to characterize the behavior above threshold, we have preferred to focus our efforts on larger $g$ values, where the asymptotic regime is much less dependent on the selection of the initial condition.

A qualitative instance of the collective dynamics can be appreciated in Fig. 5, where $R(t)$ and $Y(t)$ are plotted for $g=1.3$, showing that the evolution is more complex than just periodic.

A more quantitative characterization of the collective temporal behavior can be obtained by looking at power spectra. The square amplitude of the Fourier transform of $Y(t)$ is reported in Fig. 6 for two different coupling strengths.

We see that the spectra possess quite a rich structure, being neither trivially broadband nor just revealing a periodic behavior (especially for $g=0.8$ ). A closer look at the width of the various peaks upon increasing the network size reveals that they do not decrease. Simulations performed for different realizations of the bare frequencies (data not shown) indicate that the results are almost independent, especially for the larger system sizes. We are thus led to conclude that the stochasticlike dynamics is not due to finite-size effects, but it is intrinsic of the thermodynamic limit.

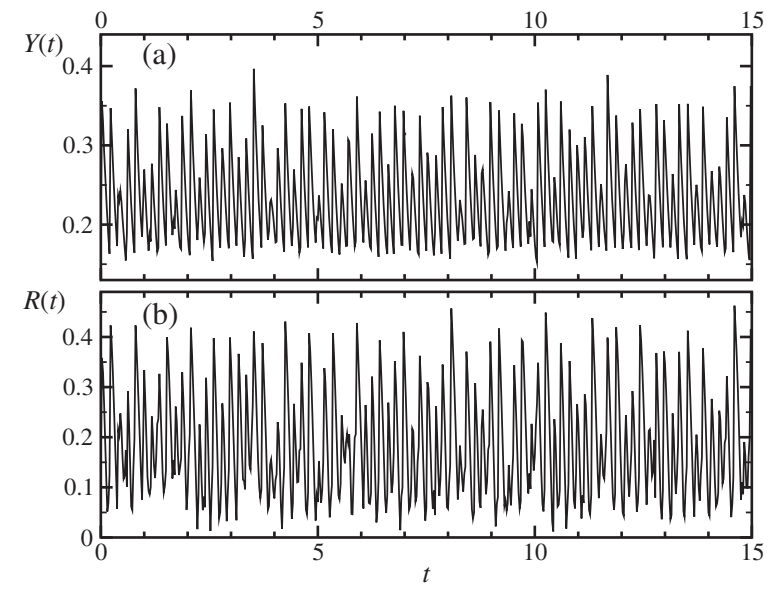

FIG. 5. Time evaluation of $Y$ (a) and $R$ (b) for $g=1.3$ and system sizes $N=4000$.

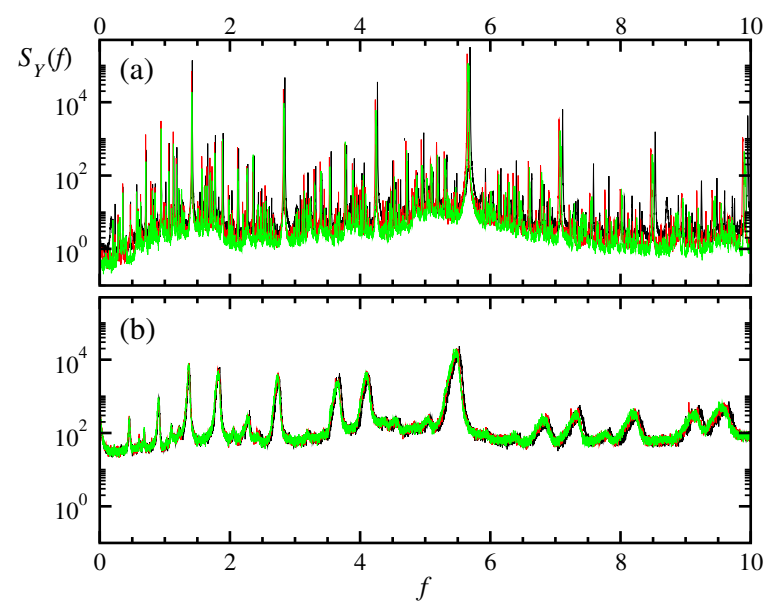

FIG. 6. Power spectra of $Y$ for $g=0.8$ (a) and 1.3 (b) in each case for $N=4000$ (black), $N=16000$ (red), and $N=64000$ (green). The spectra are obtained by transforming time series of 819.175 time units, sampled every 0.025 units and averaged over 50 different realizations.

According to the theory of nonlinear dynamical systems, it is well known that an irregular evolution may well be the manifestation of low-dimensional deterministic chaos. Can it be the case here? In order to clarify the point, it is natural to investigate the behavior of the activity field by performing a nonlinear time series analysis to determine its fractal dimension. Given a time series $Y\left(t_{n}\right)$, sampled at equally spaced times $\left(\Delta t=t_{n+1}-t_{n}=0.025\right)$, one starts embedding the series into a space of dimension $m$, by building vectors of the type $\left[Y\left(t_{n}\right), Y\left(t_{n+s}\right), \ldots, Y\left(t_{n+(m-1) s}\right)\right]$, where $s$ is suitably selected. As often done, we have chosen $s$, so that $s \Delta t$ is close to the first minimum of the autocorrelation of $Y(t)(s=5$, in our case).

The fractal dimension has then been estimated by using the nearest-neighbor method [49], as it suffers from less fluctuations in the region of small distances. Given a generic time series, $N_{r}$ reference points are randomly selected $\left(N_{r}=10^{5}\right.$ in our case). Each of them is compared with an increasing number $n$ of randomly selected measurement points (the other points in the time series-up to a maximum $N_{m}=16 \times 10^{6}$ ), monitoring the distance $\varepsilon_{m}(k, n)$ of the $k$ th neighbor (the distance is herein estimated using the maximum norm), for different values of the embedding dimension $m$ and $k$. A well-established theory [49] implies that, for large $n$,

$$
-\left\langle\ln \varepsilon_{m}(k, n)\right\rangle \approx \frac{\ln n}{D_{e}}
$$

where the angular brackets denote the average over the reference points, while $D_{e}$ is the (effective) information dimension. In order to make the dependence of $D_{e}$ on the resolution $\varepsilon_{m}$ transparent, we have modified the standard approach. After interpreting the logarithmic derivative of $n$ as a resolution-dependent dimension, 


$$
D_{e}\left(\varepsilon_{m}\right)=-\frac{d \ln n}{d\left\langle\ln \varepsilon_{m}\right\rangle}
$$

we have plotted it versus $\left\langle\varepsilon_{m}\right\rangle$ itself and interpreted it as an independent variable. In fact, we have verified that $D_{e}\left(\varepsilon_{m}\right)$ takes the same value, irrespective of the way $\varepsilon_{m}$ has been determined (i.e., independently of the $k$ value). The only differences are that larger $k$ values yield smaller statistical fluctuations, but they are confined to larger distances. A good compromise has been obtained by gluing together the data obtained for the largest $k$ value (30) with the data obtained for the smallest distances and a lower-order neighbor (the fourth one).

The results for $g=0.8$ are reported in Fig. 7(a) and different ensemble sizes $(N=4000,16000$, and 64000). The first point to notice is that for the lowest embedding dimension $(m=4), D_{e}$ nicely converges to 4 upon decreasing $\varepsilon$. This clearly implies that the dimension of the collective motion is at least 4 ; i.e., one needs at least four variables to characterize such a behavior. Furthermore, the curves obtained for the larger $m$ values reveal an increase, possibly hinting to $m$, thus suggesting that the dynamics is high dimensional (if not even infinite dimensional). Additionally, one can also appreciate a small shift to smaller scales of the curves obtained for $N=64000$. In itself, this is the indication of finite-size effects. If the shift continues as such by further increasing $m$, this would mean that part of the high dimensionality is just a consequence of statistical fluctuations that disappear in the thermodynamic limit. We are more inclined to attribute such discrepancies to another type of finite-size effect: a nonperfect equivalence among the various realizations of the frequency distributions. We have, in fact, observed that different clusters may temporarily form during the evolution, especially in the interval $g \in\left[g_{c}, 1.2\right]$ (see a more detailed discussion in Sec. V).

For $g=1.3$, the convergence to the thermodynamic limit is more clear. In Fig. 7(b), the agreement among the different network sizes is compelling over a wide range,
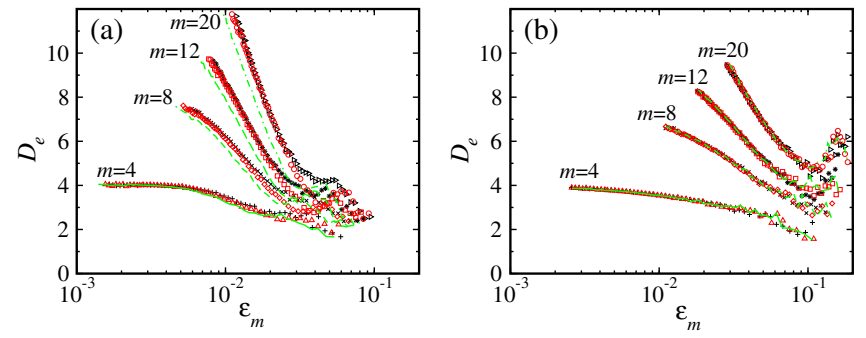

FIG. 7. Effective dimension $D_{e}$ as a function of the resolution $\varepsilon_{m}$ for $g=0.8$ and $g=1.3$ in panels (a) and (b), respectively. The system size is $N=4000$ (black), $N=16000$ (red), and $N=64000$ (green). The different symbols belong to different embedding dimensions $m$ marked in the figure. The curves for the same embedding dimension group, together with a similar slope irrespective of the system size. thus suggesting that the statistical fluctuations do not affect the dimension estimates. We have also double-checked the results by computing the correlation dimension with the TISEAN package [50]: a rather similar pattern emerges (data not shown).

Additional information is contained in the generalized order parameters. Simulations performed for $N=16000$ reveal that all of the $R_{k}$ 's depart from zero above the same critical coupling strength $g_{c}$. Additionally, several $R_{k}$ 's are significantly different from zero (actually, $R_{3}$ is the largest one). Even more interesting is that, as shown in Fig. 8, the dynamics of $R_{3}$ and $R_{1}$ are substantially uncorrelated even slightly above threshold (namely, for $g=0.7$ ). The relatively large set of $\left(R_{1}, R_{3}\right)$ pair values reveals that the knowledge of $R_{1}$ alone is not sufficient to characterize the collective dynamics.

Finally, we have investigated the degree of (in)stability of the dynamics, computing the first 10 Lyapunov exponents $\Lambda_{j}$. We have followed the approach described in Ref. [51], which consists in formally interpreting the time evolution as a series of discrete-time maps from one to the next spike emission. The results are plotted in Fig. 4(c) upon varying the coupling strength. We see that the dynamics is always stable (notice that the zero Lyapunov exponent, always present in a nonconstant autonomous dynamics, is automatically discarded). For a vanishing $g$, all the Lyapunov exponents converge to zero: This is obvious since in this limit, all the oscillators are uncoupled. Much less trivial is that the Lyapunov exponents are all negative in spite of a dynamics that may even be collectively irregular. This manifestation of stable chaos strongly suggests that the connection between different levels of descriptions (microscopic vs macroscopic) of a given model is weak, if it exists at all.

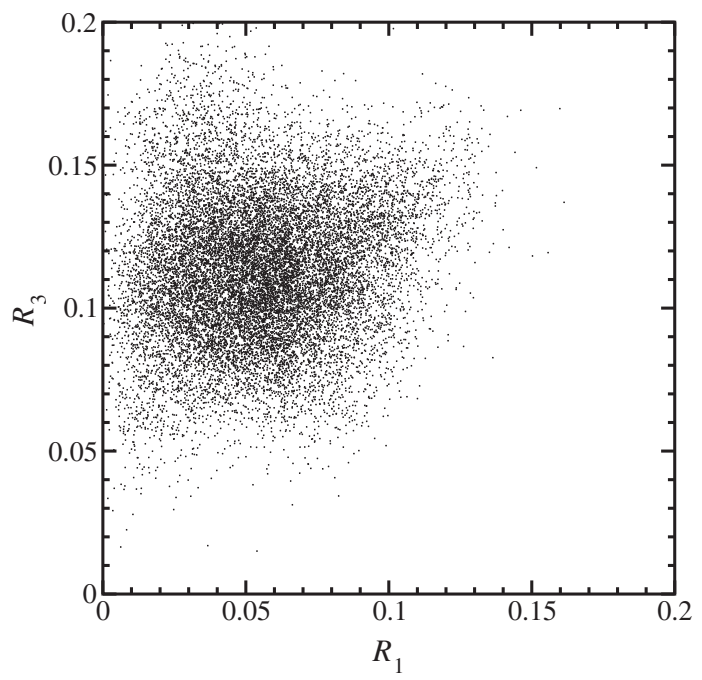

FIG. 8. Projection of the collective dynamics on the plane $\left(R_{1}, R_{3}\right)$ for $g=0.7$ in an ensemble of 16000 oscillators. The dots show 16384 time-discrete $R_{1}, R_{3}$ pairs sampled every 2.5 time units. 


\section{MICROSCOPIC DYNAMICS}

In this section, we try to shed light on the collective dynamics by analyzing the behavior of the single neurons. We notice that the coupling modifies the firing rate of the neurons. This can be appreciated in Fig. 9(a), where the effective (average) frequency $\tilde{\omega}$ is reported for the coupling strength $g=1$. The dashed line corresponds to the bare frequency of each neuron. Almost everywhere, $\tilde{\omega}$ is smaller than the bare frequency $\omega$. This is a consequence of the fact that, although the PRC was chosen to be symmetric around zero, this is no longer true for the effective PRC (see Fig. 1). The most interesting feature to notice is, however, the staircase structure of $\tilde{\omega}(\omega)$ with flat plateaus that correspond to clusters of mutually synchronized neurons: The synchronization does not mean a perfect phase locking but that the phase differences never become larger than $2 \pi$.

One way to characterize the irregularity of the singleneuron activity is through its coefficient of variation $(\mathrm{CV})$,
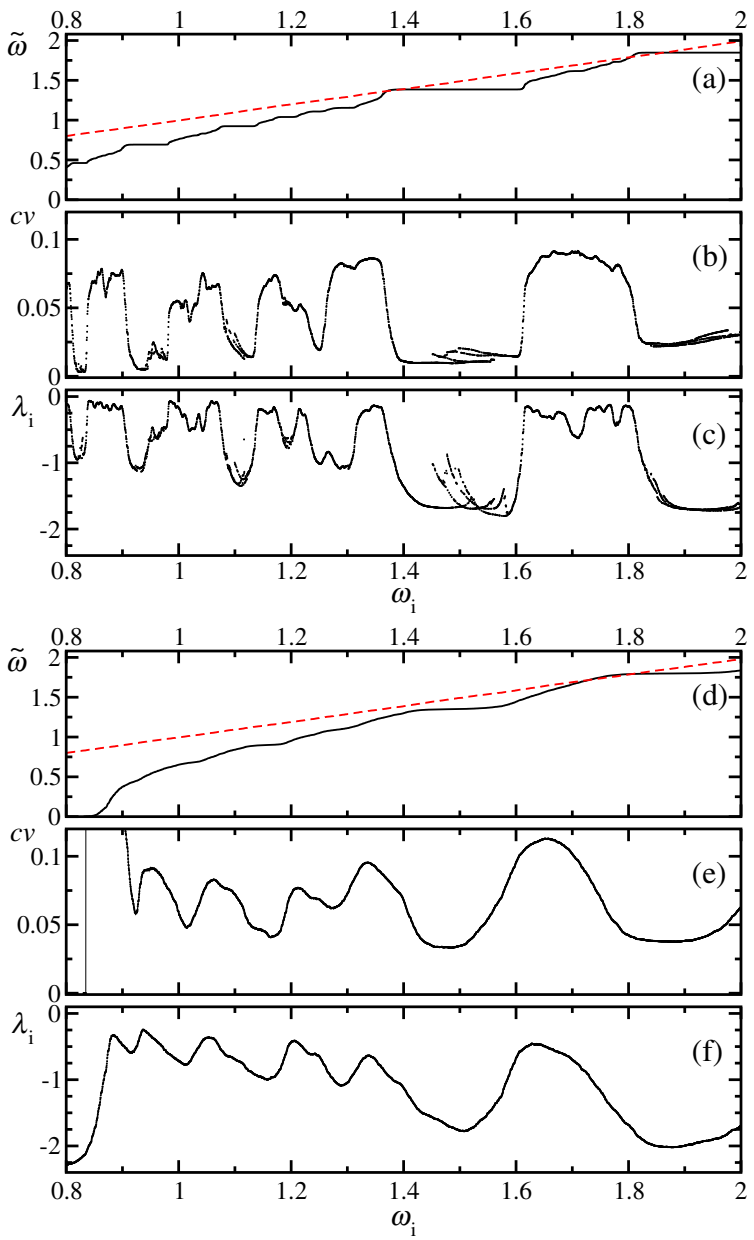

FIG. 9. Effective frequency $\tilde{\omega}$ [panels (a) and (d)], CV of $\tilde{\omega}$ [panels (b) and (e)], and conditional Lyapunov exponents $\lambda_{i}$ [panels (c) and (f)] for the different oscillators, for $g=1$ [panels (a)-(c)] and $g=1.3$ [panels (d)-(f)], all for $N=4000$. The red dashed line in panels (a) and (d) show the bare frequency as a reference for the effective frequency shown with solid black lines. i.e., the standard deviation of the interspike interval rescaled to its average value. In Fig. 9(b), we see that the $\mathrm{CV}$ allows us to identify synchronized clusters as those frequency intervals where the fluctuations are significantly smaller. Furthermore, distinct lines can be recognized inside some clusters: They correspond to different locked states [52] and are a manifestation of the multistability that is, in fact, also seen at the macroscopic level. On a more quantitative level, the neural dynamics is not significantly irregular if compared, for instance, to the true brain activity in the resting state. However, it should be kept in mind that in our toy model, the only source of disorder is the distribution of bare frequencies; no disorder has been assumed in the synaptic connections.

Additional information can be extracted by assuming that the self-determined activity field $E(t)$ is externally given, so each neuron can be interpreted as a forced dynamical system. In this way, it is natural to compute the (conditional) Lyapunov exponent $\lambda_{i}$. In Fig. 9(c), one can observe a scenario that is qualitatively different from what is observed in the Kuramoto model: All $\lambda_{i}$ 's are negative, including those of the neurons outside the flat plateaus. We come back to this point later in this section.

A partially different scenario is found for $g=1.3$ [see Figs. 9(d)-9(f)]. First of all, any sign of multistability has disappeared as have all plateaus. The initial high peak of the CV [panel (e)] is due to the fact that now the neurons with the lowest bare frequency do not spike at all, having undergone a kind of oscillation death. Their $\mathrm{CV}$ is obviously equal to zero. As a result, the first erratically spiking neurons are characterized by long interspike intervals and are obviously accompanied by large fluctuations. The $\mathrm{CV}$ of these rarely firing neurons is as large as 1.75 [not shown in Fig. 9(e) with the present scale]. The kind of oscillation-death phenomena could be seen as an inhomogeneous limit cycle (IHLC) because nonspiking neurons are not trapped at a steady state but, rather, move back and forth according to their bare frequency and the global pulses, never reaching the threshold [53]. Hence, we observe two groups of neurons, the quiet neurons, which fluctuate below threshold, and the firing neurons. The situation is a more complicated than the usual IHLC because of the many (in the thermodynamic limit, infinitely many) frequencies reflected in the power spectra (Fig. 6). Moreover, the dynamics of each oscillator is rather stable.

Altogether, the microscopic analysis confirms that the microscopic behavior is linearly stable: Each neuron is synchronized with the self-generated mean field $E(t)$, and yet an irregular dynamics is self-sustained. This can be understood by noticing that in many frequency ranges, the effective frequency is a strictly monotonous function of $\omega$. This means that, even though each neuron synchronizes with the field $E$, the parameter (bare frequency) mismatch induces a qualitatively different response: Such qualitative differences are then responsible for the maintenance of the 
self-generated irregularity. In a more technical way, the response of a phase oscillator is not structurally stable: One can slightly modify its frequency and still observe significant changes (phase slips). A more physical (although still qualitative) way to understand the phenomenon is as follows: One can see each phase oscillator as a particle moving in a potential with an inclination that depends on its bare frequency and the mean field $E$. When two particles with slightly different $\omega$ are followed, one of them may be blocked in a (shallow) minimum that is absent for the other.

One can learn a bit more about the dependence on $\omega$ by comparing pairs of consecutive oscillators (consecutive in the space of bare frequencies). This can be done by monitoring phase slips, i.e., the time instants when the phase difference becomes larger (smaller) than $1 / 2(-1 / 2)$. Since it is possible that the phase difference will oscillate around $1 / 2(-1 / 2)$, yielding long sequences of positive and negative slips, we have chosen to record only those events where two or more consecutive positive (negative) flips are observed.

In Fig. 10(a), we see that for $g=1$, there exist totally empty bands: They correspond to the previously mentioned synchronization areas. In panel (b) (which corresponds to $g=1.3$ ), both forward and backward slips are simultaneously present. One can see that the phase slips happen on a much longer time scale than the interspike intervals. Furthermore, the bands with sparse phase slips observed for $g=1.3$ are reminiscent of the synchronization bands found for $g=1.0$ : There, phase-slip events are rare and erratic. The totally empty band at the bottom frequencies for $g=1.3$ corresponds to the nonfiring neurons.

In the thermodynamic limit, the most appropriate way to characterize the collective dynamics is by monitoring the probability distribution $Q(\phi, \omega, t)$ introduced in Sec. III. In Fig. 11, we give an idea of the way it looks below and above threshold at some randomly chosen time. In panel (a), one can recognize a reasonably smooth distribution. In fact, for $g=0.5$, we are in the asynchronous regime and thereby expect a smooth distribution of the phases $\phi$ [54]. Such a distribution loses stability above $g_{c}$.

In fact, for $g=1.3$, we see a rather different structure [see panel (b)] characterized by an alternation of highly
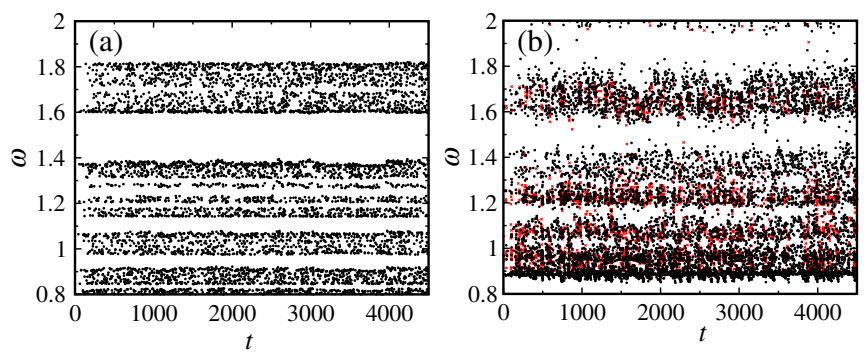

FIG. 10. Phase slips for $g=1$ [panel (a)] and $g=1.3$ [panel (b)] for $N=4000$. Black circles correspond to forward slips; red crosses correspond to backward slips, occurring only in panel (b).
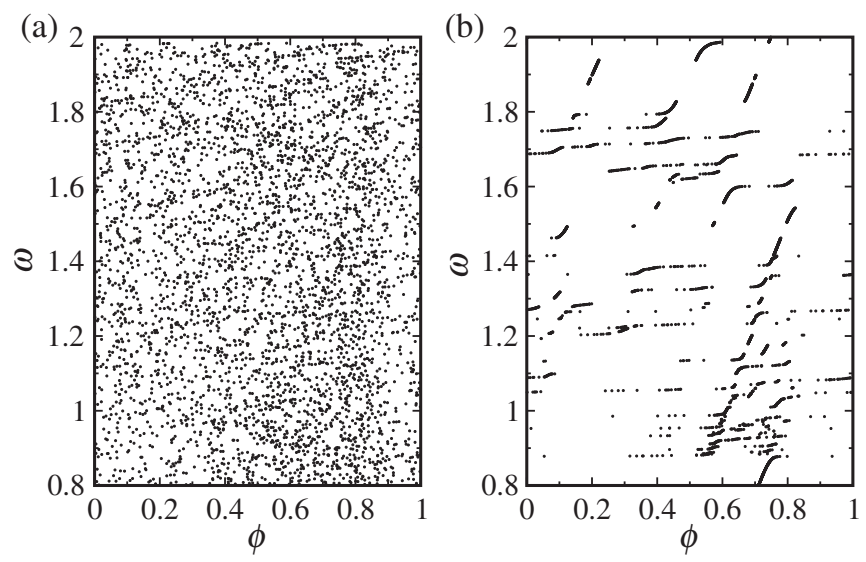

FIG. 11. Snapshots of the probability density $Q(\phi, \omega, t)$ for $g=0.5$ [panel (a)] and $g=1.3$ [panel (b)] and $N=4000$.

dense and widely spread regions. It is clear that even the plain integration of Eqs. (4) and (5) is a highly nontrivial task, not to mention the development of approximate analytical schemes.

\section{DISCUSSION AND OPEN PROBLEMS}

In this paper, we have analyzed an ensemble of pulsecoupled oscillators characterized by a distribution of bare frequencies and coupled through a homogeneous mean field. Although the setup is reminiscent of the Kuramoto model, the collective dynamics is much richer and accompanied by a linearly stable microscopic dynamics.

A linear stability analysis of the asynchronous regime allows us to identify the transition point beyond which a complex form of synchronization sets in. A numerical analysis of a properly defined Kuramoto order parameter $R$ and of the smoothed activity field $Y$ reveals that they not only fluctuate in time but that their behavior involves a large (possibly infinite) number of degrees of freedom. This indicates that even in "simple" mean-field models, such as the one investigated in this paper, the coarse-grained activity of an ensemble of phase oscillators cannot be reduced to the evolution of one or a few variables, such as the firing rate and related observables. In principle, nothing prevents a population of phase oscillators from selfsustaining a macroscopic irregular dynamics: The corresponding evolution equation is indeed a nonlinear functional equation [see Eqs. (4) and (5)], which operates in an infinite-dimensional phase space. However, it is unclear under which conditions many degrees of freedom can be simultaneously active. In order to make further progress, it will be necessary to find suitable approximations of the probability density $Q(\phi, \omega, t)$ : This task seems to require clever ideas on the way to expand $Q(\phi, \omega, t)$. One question is particularly relevant: whether the dynamics is high dimensional from the very beginning (such as in models of balanced states $[55,56])$ or whether the complexity 
increases by undergoing a series of consecutive bifurcations. The numerical analysis in the vicinity of the critical point is affected by too-strong finite-size corrections to be able to draw any conclusion.

Another open point is the generality of this scenario. Several preliminary simulations performed with various choices of the PRC reveal that it is quite robust, although the presence of a relatively steep branch seems to be a necessary condition. This is not too serious a limitation, as it naturally appears in systems characterized by a slow-fast dynamics (see the discussion in Ref. [57]). However, it might be worth assuming a different PRC shape to enable deeper analytical studies. We have indeed derived a very general equation for the loss of stability of the asynchronous state: If one could go beyond this, including the most relevant nonlinear terms, it should be possible to decide how many degrees of freedom are switched on.

Our numerical studies suggest that the transition disappears when the distribution of frequencies is narrow enough, but this is by no means a proof: Understanding whether it is strictly necessary to go beyond the weakcoupling, weak-disorder limit is another point that will be worth exploring. In this perspective, it is worth noticing that the system discussed in this paper [Eq. (1)] is an instance of a Winfree model [58] in the limit of a $\delta$-like coupling strength. This model of phase oscillators, introduced in 1967, and almost forgotten for several years, was later reconsidered for the rich phenomenology it may exhibit. However, all the variants explored in the literature so far (see, e.g., Refs. [32,59]) have not exhibited the complex collective dynamics described in this manuscript. Once again, identifying the structure of the coupling function is a crucial objective for the next investigations.

Another intriguing property of the collective dynamics discussed in this paper is the presence of a spectrum of negative Lyapunov exponents. This means that it is a manifestation of stable chaos [33]. Within the context of computational neuroscience, the stability of the microscopic trajectories suggests that this model is a good candidate for performing computational tasks. It will be worth exploring this opportunity by studying the response of this type of network to different classes of external stimuli.

\section{ACKNOWLEDGMENTS}

A.P. wishes to acknowledge a discussion with $\mathrm{M}$. Wolfrum on the stability of the Kuramoto model. This work is partially supported by the ITN-EJD COSMOS (No. 642563) project.

\section{APPENDIX A: STABILITY ANALYSIS}

Equation (9) for $u$ can be rewritten as

$$
\frac{d u}{d \phi}=\frac{\left(g \Gamma^{\prime}(\phi) E_{0}-\mu\right) u+g\left[\Gamma^{\prime}(\phi) Q_{0}+\Gamma(\phi) \frac{d Q_{0}}{d \phi}\right] z}{\omega-g \Gamma(\phi) E_{0}},
$$

which has the structure

$$
\frac{d u}{d \phi}=(A-\mu \tau) u+g P(\omega) C(\phi, \omega) z,
$$

where

$$
\begin{gathered}
A=\frac{g \Gamma^{\prime}(\phi) E_{0}}{\omega-g \Gamma(\phi) E_{0}}, \\
C(\phi, \omega)=\frac{\omega \Gamma^{\prime}(\phi)}{T\left(\omega, E_{0}\right)\left(\omega-g \Gamma(\phi) E_{0}\right)^{3}},
\end{gathered}
$$

while $\tau$ is defined in Eq. (7). The general solution of Eq. (A1) is

$$
\begin{aligned}
u(\phi, \omega)= & \mathrm{e}^{F(\phi, \omega)-\mu T(\phi, \omega)}[u(0, \omega) \\
& \left.+z \int_{0}^{\phi} d \psi C(\psi, \omega) \mathrm{e}^{-F(\psi, \omega)+\mu T(\psi, \omega)}\right],
\end{aligned}
$$

where

$$
F(\psi, \omega)=\int_{0}^{\psi} d \eta A(\eta)=\log \frac{\omega-g \Gamma(0) E_{0}}{\omega-g \Gamma(\psi) E_{0}} .
$$

Notice that $F(1)=F(0)=0$, while $T(\psi, \omega)$ is defined in Eq. (7). One can therefore write the solution $u(\phi, \omega)$ as

$$
\begin{aligned}
u(\phi, \omega)= & \frac{\omega-g \Gamma(0) E_{0}}{\omega-g \Gamma(\phi) E_{0}} \mathrm{e}^{-\mu T(\phi, \omega)}[u(0, \omega) \\
& \left.+z g P(\omega) \frac{V_{\mu}(\phi, \omega)}{\omega-g \Gamma(0) E_{0}}\right]
\end{aligned}
$$

where

$$
V_{\mu}(\phi, \omega)=\frac{\omega}{T(1, \omega)} \int_{0}^{\phi} d \psi \frac{\Gamma^{\prime}(\psi) \mathrm{e}^{\mu(\psi, \omega)}}{\left(\omega-g \Gamma(\psi) E_{0}\right)^{2}} .
$$

By imposing the periodicity condition $u(1, \omega)=u(0, \omega)$, one obtains

$$
u(1, \omega)=g z \frac{P(\omega) V_{\mu}(1, \omega)(1, \omega)}{\left(\mathrm{e}^{\mu T(1, \omega)}-1\right)\left(\omega-g \Gamma(0) E_{0}\right)} .
$$

Finally, recalling the definition of $z$ in Eq. (10), it is possible to rewrite Eq. (A8) as the eigenvalue equation

$$
1+g \Gamma(1) \int Q_{0}(1, \omega) d \omega=g \int d \omega \frac{P(\omega) V_{\mu}(1, \omega)}{\mathrm{e}^{\mu T(1, \omega)}-1} .
$$




\section{APPENDIX B: TRANSFORMATION TO THE APPROPRIATE KURAMOTO-LIKE PHASE $\vartheta$}

We solve the original system Eq. (1) with the PRC Eq. (2) in terms of the nonhomogeneously advancing phase $\phi$. At each time point that we calculate the Kuramoto order parameter $R$, we convert the phase $\phi$ into the Kuramoto-like phase $\vartheta$ according to Eqs. (12) and (13). For simplicity and readability, we introduce new constants similar to those defining the piecewise linear PRC Eq. (2): $\mathfrak{B}_{01}=$ $(g / N) E_{0} B_{01}, \quad \mathfrak{B}_{02}=(g / N) E_{0} B_{02}, \quad \mathfrak{B}_{03}=(g / N) E_{0} B_{03}$, $\mathfrak{b}_{1}=(g / N) E_{0} b_{1}$, and $\mathfrak{b}_{2}=(g / N) E_{0} b_{2}$. Hence, the Kuramoto-like phase is

$$
\vartheta(\phi(t))= \begin{cases}\frac{\tilde{\omega}}{\mathfrak{b}_{1}} \ln \frac{\omega-\mathfrak{B}_{01}}{\omega-\mathfrak{B}_{01}-\mathfrak{b}_{1} \phi(t)} & \text { if } 0 \leq \phi<\phi_{l} \\ \vartheta_{l}-\frac{\tilde{\omega}}{\mathfrak{b}_{2}} \ln \frac{\omega-\mathfrak{B}_{02}+\mathfrak{b}_{2} \phi_{l}}{\omega-\mathfrak{B}_{02}+\mathfrak{b}_{2} \phi(t)} & \text { if } \phi_{l} \leq \phi \leq \phi_{r} \\ \vartheta_{r}+\frac{\tilde{\omega}}{\mathfrak{b}_{1}} \ln \frac{\omega-\mathfrak{B}_{03}-\mathfrak{b}_{1} \phi_{r}}{\omega-\mathfrak{B}_{03}-\mathfrak{b}_{1} \phi(t)} & \text { if } \phi_{r}<\phi<1,\end{cases}
$$

with the field $E_{0}$ as stated in Eq. (8) and the effective frequency defined as the inverse interspike interval, i.e., $\tilde{\omega}=1 / T\left(1, \omega, E_{0}\right)[$ Eq. (7)]. The interspike interval can be expressed explicitly for the given PRC:

$$
\begin{aligned}
T\left(1, \omega, E_{0}\right)= & \frac{1}{\mathfrak{b}_{1}} \ln \frac{\omega-\mathfrak{B}_{01}}{\omega-\mathfrak{B}_{01}-\mathfrak{b}_{1} \phi_{l}} \\
& -\frac{1}{\mathfrak{b}_{2}} \ln \frac{\omega-\mathfrak{B}_{02}+\mathfrak{b}_{2} \phi_{l}}{\omega-\mathfrak{B}_{02}+\mathfrak{b}_{2} \phi_{r}} \\
& +\frac{1}{\mathfrak{b}_{1}} \ln \frac{\omega-\mathfrak{B}_{03}-\mathfrak{b}_{1} \phi_{r}}{\omega-\mathfrak{B}_{03}-\mathfrak{b}_{1}} .
\end{aligned}
$$

As shown in Fig. 1, the transitions $\vartheta_{l}$ and $\vartheta_{r}$ in the effective PRC $\tilde{\Gamma}$ depend on the bare frequency $\omega$ according to

$$
\begin{aligned}
\vartheta_{l} & =\frac{\tilde{\omega}}{\mathfrak{b}_{1}} \ln \frac{\omega-\mathfrak{B}_{01}}{\omega-\mathfrak{B}_{01}-\mathfrak{b}_{1} \phi_{l}}, \\
\vartheta_{r} & =\vartheta_{l}-\frac{\tilde{\omega}}{\mathfrak{b}_{2}} \ln \frac{\omega-\mathfrak{B}_{02}+\mathfrak{b}_{2} \phi_{l}}{\omega-\mathfrak{B}_{02}+\mathfrak{b}_{2} \phi_{r}} .
\end{aligned}
$$

[1] W. Gerstner and W. Kistler, Spiking Neuron Models: An Introduction (Cambridge University Press, New York, 2002).

[2] C. van Vreeswijk, Partial Synchronization in Populations of Pulse-Coupled Oscillators, Phys. Rev. E 54, 5522 (1996).

[3] A. Politi and M. Rosenblum, Equivalence of PhaseOscillator and Integrate-and-Fire Models, Phys. Rev. E 91, 042916 (2015).

[4] D. Hansel, G. Mato, and C. Meunier, Synchrony in Excitatory Neural Networks, Neural Comput. 7, 307 (1995).
[5] H. Daido, Order Function and Macroscopic Mutual Entrainment in Uniformly Coupled Limit-Cycle Oscillators, Prog. Theor. Phys. 88, 1213 (1992); Critical Conditions of Macroscopic Mutual Entrainment in Uniformly Coupled Limit-Cycle Oscillators, Prog. Theor. Phys. 89, 929 (1993); Onset of Cooperative Entrainment in LimitCycle Oscillators with Uniform All-to-All Interactions: Bifurcation of the Order Function, Physica (Amsterdam) 91D, 24 (1996).

[6] H. R. Wilson and J. D. Cowan, Excitatory and Inhibitory Interactions in Localized Populations of Model Neurons, Biophys. J. 12, 1 (1972).

[7] S. Coombes, Waves, Bumps, and Patterns in Neural Field Theories, Biol. Cybern. 93, 91 (2005).

[8] B. G. B. Ermentrout and D. H. Terman, Mathematical Foundations of Neuroscience (Springer, New York, 2010), Vol. 35.

[9] T. B. Luke, E. Barreto, and P. So, Complete Classification of the Macroscopic Behavior of a Heterogeneous Network of Theta Neuron, Neural Comput. 25, 3207 (2013).

[10] P. So, T. B. Luke, and E. Barreto, Networks of Theta Neurons with Time-Varying Excitability: Macroscopic Chaos, Multistability, and Final-State Uncertainty, Physica (Amsterdam) 267D, 16 (2014).

[11] C. R. Laing, Derivation of a Neural Field Model from a Network of Theta Neurons, Phys. Rev. E 90, 010901 (2014).

[12] E. Montbrió, D. Pazó, and A. Roxin, Macroscopic Description for Networks of Spiking Neurons, Phys. Rev. X 5, 021028 (2015).

[13] E. Ott and T. M. Antonsen, Low Dmensional Behavior of Large Systems of Globally Coupled Oscillators, Chaos 18 , 037113 (2008).

[14] S. Watanabe and S. H. Strogatz, Constants of Motion for Superconducting Josephson Arrays, Physica (Amsterdam) 74D, 197 (1994).

[15] A. Arieli, D. Shoham, R. Hildesheim, and A. Grinvald, Coherent Spatiotemporal Patterns of Ongoing Activity Revealed by Real-Time Optical Imaging Coupled with Single-Unit Recording in the Cat Visual Cortex, J. Neurophysiol. 73, 2072 (1995).

[16] M. Tsodyks, T. Kenet, A. Grinvald, and A. Arieli, Linking Spontaneous Activity of Single Cortical Neurons and the Underlying Functional Architecture, Science 286, 1943 (1999).

[17] A. Destexhe, Intracellular and Computational Evidence for a Dominant Role of Internal Network Activity in Cortical Computations, Curr. Opin. Neurobiol. 21, 717 (2011).

[18] M. N. Shadlen and W. T. Newsome, The Variable Discharge of Cortical Neurons: Implications for Connectivity, Computation, and Information Coding, J. Neurosci. 18, 3870 (1998).

[19] D. A. Butts, C. Weng, J. Jin, C.-I. Yeh, N. A. Lesica, J.-M. Alonso, and G. B. Stanley, Temporal Precision in the Neural Code and the Timescales of Natural Vision, Nature (London) 449, 92 (2007).

[20] C. van Vreeswijk and H. Sompolinsky, Chaos in Neuronal Networks with Balanced Excitatory and Inhibitory Activity, Science 274, 1724 (1996). 
[21] C. van Vreeswijk and H. Sompolinsky, Chaotic Balanced State in a Model of Cortical Circuits, Neural Comput. 10, 1321 (1998).

[22] T. Shibata, T. Chawanya, and K. Kaneko, Noiseless Collective Motion out of Noisy Chaos, Phys. Rev. Lett. 82, 4424 (1999).

[23] K. A. Takeuchi and H. Chaté, Collective Lyapunov Modes, J. Phys. A 46, 254007 (2013).

[24] S. Luccioli and A. Politi, Irregular Collective Behavior of Heterogeneous Neural Networks, Phys. Rev. Lett. 105, 158104 (2010).

[25] Y. Kuramoto, Chemical Oscillations, Waves and Turbulence (Springer, Berlin, 1984).

[26] J. A. Acebron, L. L. Bonilla, C. J. Perez Vicente, F. Ritort, and R. Spigler, The Kuramoto Model: A Simple Paradigm for Synchronization Phenomena, Rev. Mod. Phys. 77, 137 (2005).

[27] Per S. Skardal, E. Ott, and J. G. Restrepo, Cluster Synchrony in Systems of Coupled Phase Oscillators with Higher-Order Coupling, Phys. Rev. E 84, 036208 (2011).

[28] M. Komarov and A. Pikovsky, The Kuramoto Model of Coupled Oscillators with a Bi-Harmonic Coupling Function, Physica (Amsterdam) 289D, 18 (2014).

[29] P. Ashwin and O. Burylko, Weak Chimeras in Minimal Networks of Coupled Phase Oscillators, Chaos 25, 013106 (2015).

[30] H. Daido, Multi-Branch Entrainment and Multi-Peaked Order-Functions in a Phase Model of Limit-Cycle Oscillators with Uniform All-to-All Coupling, J. Phys. A 28, L151 (1995).

[31] H. Daido, Multibranch Entrainment and Scaling in Large Populations of Coupled Oscillators, Phys. Rev. Lett. 77, 1406 (1996).

[32] D. Pazó and E. Montbrió, Low-Dimensional Dynamics of Populations of Pulse-Coupled Oscillators, Phys. Rev. X 4, 011009 (2014).

[33] A. Politi and A. Torcini, Stable Chaos, in Nonlinear Dynamics and Chaos: Advances and Perspectives, Understanding Complex Systems (Springer, Berlin, 2010), pp. $103-129$.

[34] R. Zillmer, R. Livi, A. Politi, and A. Torcini, Desynchronization in Diluted Neural Networks, Phys. Rev. E 74, 036203 (2006).

[35] S. Jahnke, R.-M. Memmesheimer, and M. Timme, Stable Irregular Dynamics in Complex Neural Networks, Phys. Rev. Lett. 100, 048102 (2008).

[36] R. Zillmer, N. Brunel, and D. Hansel, Very Long Transients, Irregular Firing, and Chaotic Dynamics in Networks of Randomly Connected Inhibitory Integrate-and-Fire Neurons, Phys. Rev. E 79, 031909 (2009).

[37] M. Monteforte and F. Wolf, Dynamical Entropy Production in Spiking Neuron Networks in the Balanced State, Phys. Rev. Lett. 105, 268104 (2010).

[38] M. Monteforte and F. Wolf, Dynamic Flux Tubes Form Reservoirs of Stability in Neuronal Circuits, Phys. Rev. X 2, 041007 (2012).

[39] L. F. Abbott and C. van Vreeswijk, Asynchronous States in Networks of Pulse-Coupled Oscillators, Phys. Rev. E 48, 1483 (1993).
[40] S. H. Strogatz and R. E. Mirollo, Stability of Incoherence in a Population of Coupled Oscillators, J. Stat. Phys. 63, 613 (1991).

[41] S. H. Strogatz, R. E. Mirollo, and P. C. Matthews, Coupled Nonlinear Oscillators below the Synchronization Threshold: Relaxation by Generalized Landau Damping, Phys. Rev. Lett. 68, 2730 (1992).

[42] H. Chiba, A Proof of the Kuramoto Conjecture for a Bifurcation Structure of the Infinite-Dimensional Kuramoto Model, Ergod.Theory Dyn. Syst. 35, 762 (2015).

[43] B. Fernandez, D. Gérard-Varet, and G. Giacomin, Landau Damping in the Kuramoto Model, arXiv:1410.6006.

[44] H. Dietert, Stability and Bifurcation for the Kuramoto Model, arXiv:1411.3752v2.

[45] M. K. Stephen Yeung and S. H. Strogatz, Time Delay in the Kuramoto Model of Coupled Oscillators, Phys. Rev. Lett. 82, 648 (1999).

[46] M. Y. Choi, H. J. Kim, D. Kim, and H. Hong, Synchronization in a System of Globally Coupled Oscillators with Time Delay, Phys. Rev. E 61, 371 (2000).

[47] G. B. Ermentrout and N. Kopell, Multiple Pulse Interactions and Averaging in Systems of Coupled Neural Oscillators, J. Math. Biol. 29, 195 (1991).

[48] F. Cecconi, R. Livi, and A. Politi, Fuzzy Transition Region in a One-Dimensional Coupled-Stable-Map Lattice, Phys. Rev. E 57, 2703 (1998).

[49] R. Badii and A. Politi, Statistical Description of Chaotic Attractors: The Dimension Function, J. Stat. Phys. 40, 725 (1985).

[50] R. Hegger, H. Kantz, and T. Schreiber, Practical Implementation of Nonlinear Time Series Methods: The TISEAN Package, Chaos 9, 413 (1999).

[51] S. Olmi, R. Livi, A. Politi, and A. Torcini, Collective Oscillations in Disordered Neural Networks, Phys. Rev. E 81, 046119 (2010).

[52] As stated previously, they are not truly phase locked since the phase difference fluctuates.

[53] E. Ullner, A. Zaikin, E. I. Volkov, and J. García-Ojalvo, Multistability and Clustering in a Population of Synthetic Genetic Oscillators via Phase-Repulsive Cell-to-Cell Communication, Phys. Rev. Lett. 99, 148103 (2007); A. Koseska, E. Volkov, and J. Kurths, Oscillation Quenching Mechanisms: Amplitude vs. Oscillation Death, Phys. Rep. 531, 173 (2013).

[54] A perfect uniformity would be observed if the effective phases $\vartheta$ were used-see Appendix B for their definition.

[55] H. Sompolinsky, A. Crisanti, and H.-J. Sommers, Chaos in Random Neural Networks, Phys. Rev. Lett. 61, 259 (1988).

[56] G. Curato and A. Politi, Onset of Chaotic Dynamics in Neural Networks, Phys. Rev. E 88, 042908 (2013).

[57] P. Langfield, B. Krauskopf, and H. M. Osinga, ForwardTime and Backward-Time Isochrons and Their Interactions, SIAM J. Appl. Dyn. Syst. 14, 1418 (2015).

[58] A. T. Winfree, Biological Rhythms and the Behavior of Populations of Coupled Oscillators, J. Theor. Biol. 16, 15 (1967).

[59] Joel T. Ariaratnam and S. H. Strogatz, Phase Diagram for the Winfree Model of Coupled Nonlinear Oscillators, Phys. Rev. Lett. 86, 4278 (2001). 